\title{
Enhanced wormhole optimizer algorithm for solving optimal reactive power problem
}

\author{
Kanagasabai Lenin \\ Department of EEE, Prasad V. Potluri Siddhartha Institute of Technology, India
}

\begin{tabular}{l}
\hline Article Info \\
\hline Article history: \\
Received Nov 11, 2019 \\
Revised Nov 13, 2019 \\
Accepted Dec 27, 2019 \\
\hline
\end{tabular}

Keywords:

Enhanced wormhole optimizer Optimal reactive power

Transmission loss

\begin{abstract}
In this paper Enhanced Wormhole Optimizer (EWO) algorithm is used to solve optimal reactive power problem. Proposed algorithm based on the Wormholes which exploits the exploration space. Between different universes objects are exchanged through white or black hole tunnels. Regardless of the inflation rate, through wormholes objects in all universes which possess high probability will shift to the most excellent universe. In the projected Enhanced Wormhole Optimizer (EWO) algorithm in order to avoid the solution to be get trapped into the local optimal solution Levy flight has been applied. Projected Enhanced Wormhole Optimizer (EWO) algorithm has been tested in standard IEEE 14, 30, 57,118,300 bus test systems and simulation results show that the EWO algorithm reduced the real power loss efficiently.
\end{abstract}

This is an open access article under the CC BY-SA license.

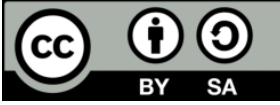

\section{Corresponding Author:}

Kanagasabai Lenin,

Department of EEE,

Prasad V. Potluri Siddhartha, Institute of Technology,

Kanuru, Vijayawada, Andhra Pradesh -520007, India.

Email: gklenin@gmail.com

\section{INTRODUCTION}

For secure and economic operations of power system optimal reactive power problem plays vital role. Several types of techniques [1-6] have been utilized to solve the problem previously. Conversely many difficulties are found while solving problem due to inequality constraints. Evolutionary techniques [7-15] are applied to solve the reactive power problem. This paper proposes Enhanced Wormhole Optimizer (EWO) algorithm for solving optimal reactive power problem. Wormhole Optimizer Algorithm is based on the Wormholes which exploit the exploration space. Wormhole tunnel are built for local change in each universe $\mathrm{m}$ through most excellent universe then probability of refinement the inflation rate is done through wormholes. Objects are exchanged through tunnels and wormholes objects which possess high probability will shift to the most excellent universe. In the projected Enhanced Wormhole Optimizer (EWO) algorithm in order to avoid the solution to be get trapped into the local optimal solution Levy flight has been applied. Projected Enhanced Wormhole Optimizer (EWO) algorithm has been tested in standard IEEE 14, 30, $57,118,300$ bus test systems and simulation results show that the projected algorithm reduced the real power loss effectively.

\section{PROBLEM FORMULATION}

Objective of the problem is to reduce the true power loss:

$$
\mathrm{F}=\mathrm{P}_{\mathrm{L}}=\sum_{\mathrm{k} \in \mathrm{Nbr}} \mathrm{g}_{\mathrm{k}}\left(\mathrm{V}_{\mathrm{i}}^{2}+\mathrm{V}_{\mathrm{j}}^{2}-2 \mathrm{~V}_{\mathrm{i}} \mathrm{V}_{\mathrm{j}} \cos \theta_{\mathrm{ij}}\right)
$$


Voltage deviation given as follows:

$$
\begin{aligned}
& \mathrm{F}=\mathrm{P}_{\mathrm{L}}+\omega_{\mathrm{v}} \times \text { Voltage Deviation } \\
& \text { Voltage Deviation } \quad=\sum_{\mathrm{i}=1}^{\mathrm{Npq}}\left|\mathrm{V}_{\mathrm{i}}-1\right|
\end{aligned}
$$

Constraint (Equality)

$$
\mathrm{P}_{\mathrm{G}}=\mathrm{P}_{\mathrm{D}}+\mathrm{P}_{\mathrm{L}}
$$

Constraints (Inequality)

$$
\begin{aligned}
& \mathrm{P}_{\text {gslack }}^{\min } \leq \mathrm{P}_{\text {gslack }} \leq \mathrm{P}_{\text {gslack }}^{\text {max }} \\
& \mathrm{Q}_{\mathrm{gi}}^{\min } \leq \mathrm{Q}_{\mathrm{gi}} \leq \mathrm{Q}_{\mathrm{gi}}^{\max }, \mathrm{i} \in \mathrm{N}_{\mathrm{g}} \\
& \mathrm{V}_{\mathrm{i}}^{\min } \leq \mathrm{V}_{\mathrm{i}} \leq \mathrm{V}_{\mathrm{i}}^{\text {max }}, \mathrm{i} \in \mathrm{N} \\
& \mathrm{T}_{\mathrm{i}}^{\min } \leq \mathrm{T}_{\mathrm{i}} \leq \mathrm{T}_{\mathrm{i}}^{\max }, \mathrm{i} \in \mathrm{N}_{\mathrm{T}} \\
& \mathrm{Q}_{\mathrm{c}}^{\min } \leq \mathrm{Q}_{\mathrm{c}} \leq \mathrm{Q}_{\mathrm{C}}^{\max }, \mathrm{i} \in \mathrm{N}_{\mathrm{C}}
\end{aligned}
$$

\section{Enhanced Wormhole Optimizer Algorithm}

Wormhole Optimizer Algorithm is based on the Wormholes which exploit the exploration space. Through wormholes objects which has high probability will shift to the most excellent universe and it modeled by using roulette wheel selection methodology as follows,

$$
U=\left[\begin{array}{ccc}
y_{11} & \cdots & y_{1 d} \\
\vdots & \ddots & \vdots \\
y_{n 1} & \cdots & y_{n d}
\end{array}\right]
$$

Number of the variables is indicated by " $\mathrm{d}$ " and number of universe which is considered as candidate solution is indicated by"n".

$$
y_{i j}= \begin{cases}y_{k j} & \text { random }_{1}<N I\left(U_{i}\right) \\ y_{i j} & \text { random }_{1}<N I\left(U_{i}\right)\end{cases}
$$

Through roulette wheel selection $y_{i j}$ 's “j”th parameter of the " $k$ "th universe will be chosen, in the "i"th universe " $\mathrm{j}$ "th parameter is expressed by $y_{k j}$, ith universe inflation rate indicated by $N I\left(U_{i}\right)$, ith universe indicated by $U_{i}$, random $_{1} \in[0,1]$.

In between two universes wormhole tunnel $[16,17]$ are built then the local change for each universe is done by most excellent universe and the elevated probability of refinement the inflation rate through wormholes is done by,

$$
y_{i j}=\left\{\begin{array}{c}
\left\{\begin{array}{c}
Y_{j}+\text { Tr. distance rate } \times\left(\left(u b_{j}-l b_{j}\right) \times \text { rand }_{4}+l b_{j}\right) \text { rand }_{3}<0.5 \\
Y_{j}-\text { Tr. distance rate } \times\left(\left(u b_{j}-l b_{j}\right) \times \text { rand }_{4}+l b_{j}\right) \text { rand }_{3} \geq 0.5 \text { rand }_{2}<w \text { e } p \\
y_{i j} \quad \text { rand }_{2} \geq \text { e e p }
\end{array}\right.
\end{array}\right.
$$

Wormhole existence probability indicated by "w e p", "tr." Indicates the travelling and random denoted by " rand".

During the optimization procedure exploitation has been enhanced as follows,

Wormhole existence probability $=\mathrm{w}_{\text {minimum }+}$ current iteration $\left(\frac{\mathrm{w}_{\text {maximum }}-\mathrm{w}_{\text {minimum }}}{\text { maximum iteration }}\right)$ 
In order to improve the local search precisely travelling distance rate will be increased over the iterations as follows,

$$
\text { Travelling distance rate }=1-\frac{\text { current iteration }^{1 / p}}{\text { maximum iteration }}{ }^{1 / p}
$$

In the projected Enhanced Wormhole Optimizer (EWO) algorithm in order to avoid the solution to be get trapped into the local optimal solution Levy flight has been applied.

Levy flight is a rank of non-Gaussian random procedure whose capricious walks are haggard from Levy stable distribution. Allocation by $L(s) \sim|s|^{-1-\beta}$ where $0<\beta<2$ is an index. Scientifically defined as,

$$
L(s, \gamma, \mu)=\left\{\begin{array}{l}
\sqrt{\frac{\gamma}{2 \pi}} \\
0 \quad \text { if } s \leq 0
\end{array} \quad \exp \left[-\frac{\gamma}{2(s-\mu)}\right] \frac{1}{(s-\mu)^{3 / 2}} \quad \text { if } 0<\mu<s<\infty\right.
$$

In terms of Fourier transform Levy distribution defined as

$$
F(k)=\exp \left[-\alpha|k|^{\beta}\right], 0<\beta \leq 2,
$$

Fresh state is calculated as,

$$
\begin{aligned}
& Y^{t+1}=Y^{t}+\alpha \oplus \operatorname{Levy}(\beta) \\
& Y^{t+1}=y^{t}+\operatorname{random}(\operatorname{size}(D)) \oplus \operatorname{Levy}(\beta)
\end{aligned}
$$

In the projected Enhanced Wormhole Optimizer (EWO) algorithm while generation of new solutions $U_{i}^{t+1}$ levy flight (y) will be applied,

$$
U_{i}^{t+1}=U_{i}^{t}+K(l b+(u b-l b) * \operatorname{levy}(y)) \times U_{i}^{t}
$$

Levy flight will be applied in the adaptive mode to balance the exploration and exploitation by applying large levy weight initially and final course the weight of the levy will be decreased,

$$
K=\left(\frac{\text { Maximum iteration-current iteration }}{\text { maximum iteration }}\right)
$$

By using Mantegna's algorithm Non-trivial scheme of engendering step size by,

$$
\begin{aligned}
& s=\frac{u}{|v|^{\frac{1}{\beta}}} \\
& Y^{t+1}=Y^{t}+\operatorname{random}(\operatorname{size}(D)) \oplus \operatorname{Levy}(\beta) \sim 0.01 \frac{u}{|v|^{1 / \beta}}\left(y_{j}^{t}-g b\right) \\
& u \sim N\left(0, \sigma_{u}^{2}\right) \quad v \sim N\left(0, \sigma_{v}^{2}\right)
\end{aligned}
$$

with

$$
\sigma_{u}=\left\{\frac{\Gamma(1+\beta) \sin (\pi \beta / 2)}{\Gamma[(1+\beta) / 2] \beta 2^{(\beta-1) / 2}}\right\}^{1 / \beta}, \sigma_{v}=1
$$

then,

$$
\operatorname{Levy}(y)=0.01 \times \frac{u \times \sigma}{|v|^{\frac{1}{\beta}}}
$$

Start

In put ; "d" \& “n” ; Lower bound $=\left[\mathrm{Lb}_{1}, \mathrm{Lb}_{2}, \ldots, ., \mathrm{L}_{\mathrm{bd}}\right]$; Upper bound $=\left[\mathrm{Ub}_{1}, \mathrm{Ub}_{2}, \ldots, \mathrm{U}_{\mathrm{bd}}\right]$; Maximum number of iterations

Output: Optimal solution

Step a: Initialization of parameters

Enhanced wormhole optimizer algorithm for solving optimal reactive ... (Kanagasabai Lenin) 
Engender arbitrary universes " $U$ " by $U P=\left\{U_{1}, U_{2}, \ldots U_{i}, \ldots, U_{n}\right\}$

Initialize Wormhole existence probability, travelling distance rate, objective function $\mathrm{t}=0$

Step b: categorization and reorganize; arrange the universes; universe inflation rate (UI) will be reorganized

Step c: Iteration; while $\mathrm{t}<$ Maximum iteration

Compute universe inflation rate; UI $\left(U_{i}^{t}\right) ; \mathrm{i}=1,2, \ldots, \mathrm{n}$

For every universe " $\mathrm{U}_{\mathrm{i}}$ "; modernize Wormhole existence probability, travelling distance rate by

Wormhole existence probability $=\mathrm{w}_{\text {minimum }+}$ current iteration $\left(\frac{\mathrm{w}_{\text {maximum }}-\mathrm{w}_{\text {minimum }}}{\text { maximum iteration }}\right)$

Travelling distance rate $=1-\frac{\text { current iteration }^{1 / p}}{\text { maximum iteration }^{1 / p}}$; Black hole index value $=\mathrm{i}$

Modernize the value " $U$ " by $U_{i}^{t+1}=U_{i}^{t}+K(l b+(u b-l b) * \operatorname{levy}(y)) \times U_{i}^{t}$

For every object $y_{i j} ;$ random $_{1}=$ random $(0,1)$;

If random $_{1}<\mathrm{UI}\left(\mathrm{U}_{\mathrm{i}}\right)$; white hole index = roulette wheel selection (-UI);

$\mathrm{U}($ black hole index, $\mathrm{j})=\mathrm{SU}($ white hole index, $\mathrm{j})$;

End if

random $_{2}=$ random $(0,1)$

If random $_{2}<$ Wormhole existence probability

random $_{3}=$ random $(0,1) ;$ random $_{4}=$ random $(0,1)$;

If random $_{3}<0.5$

$y_{i j}=$ optimal solution $(j)+$ Travelling distance rate $*\left((u b(j)-l b(j)) *\right.$ random $\left._{4}+l b(j)\right)$ Or else

$y_{i j}=$ optimal solution $(j)-$ Travelling distance rate $*\left((u b(j)-l b(j)) * \operatorname{random}_{4}+l b(j)\right)$

End if

End for

$\mathrm{t}=\mathrm{t}+1$

End while

Step d: End; output the optimal solution

\section{SIMULATION RESULTS}

At first in standard IEEE 14 bus system [18] the validity of the proposed Enhanced Wormhole Optimizer (EWO) algorithm has been tested, Table 1 shows the constraints of control variables Table 2 shows the limits of reactive power generators and comparison results are presented in Table 3.

Table 1. Constraints of control variables

\begin{tabular}{cccc}
\hline System & Variables & $\begin{array}{c}\text { Minimum } \\
(\mathrm{PU})\end{array}$ & $\begin{array}{c}\text { Maximum } \\
(\mathrm{PU})\end{array}$ \\
\hline $\begin{array}{c}\text { IEEE 14 } \\
\text { Bus }\end{array}$ & $\begin{array}{c}\text { Generator } \\
\text { Voltage } \\
\text { Transformer } \\
\text { Tap }\end{array}$ & 0.95 & 1.1 \\
& 0.9 & 1.1 \\
VAR Source & 0 & 0.20 \\
\hline
\end{tabular}

Table 2. Constrains of reactive power generators

\begin{tabular}{cccc}
\hline System & Variables & $\begin{array}{c}\text { Q Minimum } \\
\text { (PU) }\end{array}$ & $\begin{array}{c}\text { Q Maximum } \\
(\mathrm{PU})\end{array}$ \\
\hline IEEE 14 & 1 & 0 & 10 \\
Bus & 2 & -40 & 50 \\
& 3 & 0 & 40 \\
& 6 & -6 & 24 \\
& 8 & -6 & 24 \\
\hline
\end{tabular}

Table 3. Simulation results of IEEE -14 system

\begin{tabular}{|c|c|c|c|c|c|c|}
\hline Control variables & Base case & MPSO [19] & PSO [19] & EP [19] & SARGA [19] & EWO \\
\hline$V G-1$ & 1.060 & 1.100 & 1.100 & $\mathrm{NR}^{*}$ & $\mathrm{NR}^{*}$ & 1.013 \\
\hline$V G-2$ & 1.045 & 1.085 & 1.086 & 1.029 & 1.060 & 1.014 \\
\hline$V G-3$ & 1.010 & 1.055 & 1.056 & 1.016 & 1.036 & 1.002 \\
\hline$V G-6$ & 1.070 & 1.069 & 1.067 & 1.097 & 1.099 & 1.017 \\
\hline$V G-8$ & 1.090 & 1.074 & 1.060 & 1.053 & 1.078 & 1.021 \\
\hline Tap 8 & 0.978 & 1.018 & 1.019 & 1.04 & 0.95 & 0.910 \\
\hline Tap 9 & 0.969 & 0.975 & 0.988 & 0.94 & 0.95 & 0.913 \\
\hline Tap 10 & 0.932 & 1.024 & 1.008 & 1.03 & 0.96 & 0.927 \\
\hline$Q C-9$ & 0.19 & 14.64 & 0.185 & 0.18 & 0.06 & 0.120 \\
\hline$P G$ & 272.39 & 271.32 & 271.32 & $\mathrm{NR}^{*}$ & NR* & 271.78 \\
\hline$Q G$ (Mvar) & 82.44 & 75.79 & 76.79 & $\mathrm{NR}^{*}$ & NR* & 75.79 \\
\hline Reduction in PLoss (\%) & 0 & 9.2 & 9.1 & 1.5 & 2.5 & 25.85 \\
\hline Total PLoss (Mw) & 13.550 & 12.293 & 12.315 & 13.346 & 13.216 & 10.047 \\
\hline
\end{tabular}

NR* - Not reported 
Then Enhanced Wormhole Optimizer (EWO) algorithm has been tested, in IEEE 30 Bus system. Table 4 shows the constraints of control variables, Table 5 shows the limits of reactive power generators and comparison results are presented in Table 6.

Table 4. Constraints of control variables

\begin{tabular}{cccc}
\hline System & Variables & $\begin{array}{c}\text { Minimum } \\
(\mathrm{PU})\end{array}$ & $\begin{array}{c}\text { Maximum } \\
(\mathrm{PU})\end{array}$ \\
\hline IEEE 30 Bus & $\begin{array}{c}\text { Generator } \\
\text { Voltage } \\
\text { Transformer } \\
\text { Tap }\end{array}$ & 0.95 & 1.1 \\
& 0.9 & 1.1 \\
VAR Source & 0 & 0.20 \\
\hline
\end{tabular}

Table 5. Constrains of reactive power generators

\begin{tabular}{cccc}
\hline System & Variables & $\begin{array}{c}\text { Q Minimum } \\
(\mathrm{PU})\end{array}$ & $\begin{array}{c}\text { Q Maximum } \\
(\mathrm{PU})\end{array}$ \\
\hline IEEE 30 Bus & 1 & 0 & 10 \\
& 2 & -40 & 50 \\
& 5 & -40 & 40 \\
& 8 & -10 & 40 \\
& 11 & -6 & 24 \\
& 13 & -6 & 24 \\
\hline
\end{tabular}

Table 6. Simulation results of IEEE -30 system

\begin{tabular}{|c|c|c|c|c|c|c|}
\hline Control variables & Base case & MPSO [19] & PSO [19] & EP [19] & SARGA [19] & EWO \\
\hline $\mathrm{VG}-1$ & 1.060 & 1.101 & 1.100 & $\mathrm{NR}^{*}$ & $\mathrm{NR}^{*}$ & 1.013 \\
\hline $\mathrm{VG}-2$ & 1.045 & 1.086 & 1.072 & 1.097 & 1.094 & 1.014 \\
\hline $\mathrm{VG}-5$ & 1.010 & 1.047 & 1.038 & 1.049 & 1.053 & 1.010 \\
\hline $\mathrm{VG}-8$ & 1.010 & 1.057 & 1.048 & 1.033 & 1.059 & 1.021 \\
\hline $\mathrm{VG}-12$ & 1.082 & 1.048 & 1.058 & 1.092 & 1.099 & 1.032 \\
\hline VG-13 & 1.071 & 1.068 & 1.080 & 1.091 & 1.099 & 1.024 \\
\hline Tap11 & 0.978 & 0.983 & 0.987 & 1.01 & 0.99 & 0.934 \\
\hline Tap12 & 0.969 & 1.023 & 1.015 & 1.03 & 1.03 & 0.930 \\
\hline Tap15 & 0.932 & 1.020 & 1.020 & 1.07 & 0.98 & 0.921 \\
\hline Tap36 & 0.968 & 0.988 & 1.012 & 0.99 & 0.96 & 0.923 \\
\hline QC10 & 0.19 & 0.077 & 0.077 & 0.19 & 0.19 & 0.092 \\
\hline QC24 & 0.043 & 0.119 & 0.128 & 0.04 & 0.04 & 0.124 \\
\hline$P G(\mathrm{MW})$ & 300.9 & 299.54 & 299.54 & $\mathrm{NR}^{*}$ & $\mathrm{NR}^{*}$ & 297.68 \\
\hline$Q G$ (Mvar) & 133.9 & 130.83 & 130.94 & NR* & $\mathrm{NR} *$ & 131.41 \\
\hline Reduction in PLoss (\%) & 0 & 8.4 & 7.4 & 6.6 & 8.3 & 19.37 \\
\hline Total PLoss (Mw) & 17.55 & 16.07 & 16.25 & 16.38 & 16.09 & 14.149 \\
\hline
\end{tabular}

Then the proposed Enhanced Wormhole Optimizer (EWO) algorithm has been tested, in IEEE 57 Bus system. Table 7 shows the constraints of control variables, Table 8 shows the limits of reactive power generators and comparison results are presented in Table 9.

Table 7. Constraints of control variables

\begin{tabular}{cccc}
\hline System & Variables & Minimum (PU) & Maximum (PU) \\
\hline IEEE 57 Bus & Generator Voltage & 0.95 & 1.1 \\
& Transformer Tap & 0.9 & 1.1 \\
& VAR Source & 0 & 0.20 \\
\hline
\end{tabular}

Table 8. Constrains of reactive power generators

\begin{tabular}{cccc}
\hline System & Variables & Q Minimum (PU) & Q Maximum (PU) \\
\hline IEEE 57 Bus & 1 & -140 & 200 \\
& 2 & -17 & 50 \\
& 3 & -10 & 60 \\
& 6 & -8 & 25 \\
& 8 & -140 & 200 \\
& 9 & -3 & 9 \\
& 12 & -150 & 155 \\
\hline
\end{tabular}


Then the proposed Enhanced Wormhole Optimizer algorithm has been tested, in IEEE 118 Bus system. Table 10 shows the constraints of control variables and comparison results are presented in Table 11.

Table 9. Simulation results of IEEE -57 system

\begin{tabular}{|c|c|c|c|c|c|c|}
\hline Control variables & Base case & MPSO [19] & PSO [19] & CGA [19] & AGA [19] & EWO \\
\hline VG 1 & 1.040 & 1.093 & 1.083 & 0.968 & 1.027 & 1.023 \\
\hline VG 2 & 1.010 & 1.086 & 1.071 & 1.049 & 1.011 & 1.010 \\
\hline VG 3 & 0.985 & 1.056 & 1.055 & 1.056 & 1.033 & 1.034 \\
\hline VG 6 & 0.980 & 1.038 & 1.036 & 0.987 & 1.001 & 1.012 \\
\hline VG 8 & 1.005 & 1.066 & 1.059 & 1.022 & 1.051 & 1.030 \\
\hline VG 9 & 0.980 & 1.054 & 1.048 & 0.991 & 1.051 & 1.011 \\
\hline VG 12 & 1.015 & 1.054 & 1.046 & 1.004 & 1.057 & 1.040 \\
\hline Tap 19 & 0.970 & 0.975 & 0.987 & 0.920 & 1.030 & 0.952 \\
\hline Tap 20 & 0.978 & 0.982 & 0.983 & 0.920 & 1.020 & 0.937 \\
\hline Tap 31 & 1.043 & 0.975 & 0.981 & 0.970 & 1.060 & 0.920 \\
\hline Tap 35 & 1.000 & 1.025 & 1.003 & $\mathrm{NR}^{*}$ & $\mathrm{NR}^{*}$ & 1.019 \\
\hline Tap 36 & 1.000 & 1.002 & 0.985 & $\mathrm{NR}^{*}$ & $\mathrm{NR}^{*}$ & 1.007 \\
\hline Tap 37 & 1.043 & 1.007 & 1.009 & 0.900 & 0.990 & 1.009 \\
\hline Tap 41 & 0.967 & 0.994 & 1.007 & 0.910 & 1.100 & 0.990 \\
\hline Tap 46 & 0.975 & 1.013 & 1.018 & 1.100 & 0.980 & 1.010 \\
\hline Tap 54 & 0.955 & 0.988 & 0.986 & 0.940 & 1.010 & 0.971 \\
\hline Tap 58 & 0.955 & 0.979 & 0.992 & 0.950 & 1.080 & 0.966 \\
\hline Tap 59 & 0.900 & 0.983 & 0.990 & 1.030 & 0.940 & 0.963 \\
\hline Tap 65 & 0.930 & 1.015 & 0.997 & 1.090 & 0.950 & 1.001 \\
\hline Tap 66 & 0.895 & 0.975 & 0.984 & 0.900 & 1.050 & 0.950 \\
\hline Tap 71 & 0.958 & 1.020 & 0.990 & 0.900 & 0.950 & 1.001 \\
\hline Tap 73 & 0.958 & 1.001 & 0.988 & 1.000 & 1.010 & 1.000 \\
\hline Tap 76 & 0.980 & 0.979 & 0.980 & 0.960 & 0.940 & 0.968 \\
\hline Tap 80 & 0.940 & 1.002 & 1.017 & 1.000 & 1.000 & 1.002 \\
\hline QC 18 & 0.1 & 0.179 & 0.131 & 0.084 & 0.016 & 0.174 \\
\hline QC 25 & 0.059 & 0.176 & 0.144 & 0.008 & 0.015 & 0.168 \\
\hline QC 53 & 0.063 & 0.141 & 0.162 & 0.053 & 0.038 & 0.140 \\
\hline PG (MW) & 1278.6 & 1274.4 & 1274.8 & 1276 & 1275 & 1270.13 \\
\hline QG (Mvar) & 321.08 & 272.27 & 276.58 & 309.1 & 304.4 & 272.34 \\
\hline Reduction in PLoss (\%) & 0 & 15.4 & 14.1 & 9.2 & 11.6 & 24.07 \\
\hline Total PLoss (Mw) & 27.8 & 23.51 & 23.86 & 25.24 & 24.56 & 21.108 \\
\hline
\end{tabular}

Table 10. Constraints of control variables

\begin{tabular}{cccc}
\hline System & Variables & Minimum (PU) & Maximum (PU) \\
\hline IEEE 118 Bus & Generator Voltage & 0.95 & 1.1 \\
& Transformer Tap & 0.9 & 1.1 \\
& VAR Source & 0 & 0.20 \\
\hline
\end{tabular}

Table 11. Simulation results of IEEE -118 system

\begin{tabular}{ccccccc}
\hline Control variables & Base case & MPSO [19] & PSO [19] & PSO [19] & CLPSO [19] & EWO \\
\hline VG 1 & 0.955 & 1.021 & 1.019 & 1.085 & 1.033 & 1.010 \\
VG 4 & 0.998 & 1.044 & 1.038 & 1.042 & 1.055 & 1.044 \\
VG 6 & 0.990 & 1.044 & 1.044 & 1.080 & 0.975 & 1.022 \\
VG 8 & 1.015 & 1.063 & 1.039 & 0.968 & 0.966 & 1.003 \\
VG 10 & 1.050 & 1.084 & 1.040 & 1.075 & 0.981 & 1.010 \\
VG 12 & 0.990 & 1.032 & 1.029 & 1.022 & 1.009 & 1.021 \\
VG 15 & 0.970 & 1.024 & 1.020 & 1.078 & 0.978 & 1.030 \\
VG 18 & 0.973 & 1.042 & 1.016 & 1.049 & 1.079 & 1.041 \\
VG 19 & 0.962 & 1.031 & 1.015 & 1.077 & 1.080 & 1.030 \\
VG 24 & 0.992 & 1.058 & 1.033 & 1.082 & 1.028 & 1.014 \\
VG 25 & 1.050 & 1.064 & 1.059 & 0.956 & 1.030 & 1.035 \\
VG 26 & 1.015 & 1.033 & 1.049 & 1.080 & 0.987 & 1.056 \\
VG 27 & 0.968 & 1.020 & 1.021 & 1.087 & 1.015 & 0.909 \\
VG 31 & 0.967 & 1.023 & 1.012 & 0.960 & 0.961 & 0.907 \\
VG 32 & 0.963 & 1.023 & 1.018 & 1.100 & 0.985 & 0.913 \\
VG 34 & 0.984 & 1.034 & 1.023 & 0.961 & 1.015 & 1.001 \\
VG 36 & 0.980 & 1.035 & 1.014 & 1.036 & 1.084 & 1.000 \\
VG 40 & 0.970 & 1.016 & 1.015 & 1.091 & 0.983 & 0.960 \\
VG 42 & 0.985 & 1.019 & 1.015 & 0.970 & 1.051 & 1.001 \\
VG 46 & 1.005 & 1.010 & 1.017 & 1.039 & 0.975 & 1.002 \\
VG 49 & 1.025 & 1.045 & 1.030 & 1.083 & 0.983 & 1.000 \\
\hline
\end{tabular}


Table 11. Simulation results of IEEE -118 system (Continued)

\begin{tabular}{|c|c|c|c|c|c|c|}
\hline Control variables & Base case & MPSO [19] & PSO [19] & PSO [19] & CLPSO [19] & EWO \\
\hline VG 54 & 0.955 & 1.029 & 1.020 & 0.976 & 0.963 & 0.920 \\
\hline VG 55 & 0.952 & 1.031 & 1.017 & 1.010 & 0.971 & 0.961 \\
\hline VG 56 & 0.954 & 1.029 & 1.018 & 0.953 & 1.025 & 0.950 \\
\hline VG 59 & 0.985 & 1.052 & 1.042 & 0.967 & 1.000 & 0.961 \\
\hline VG 61 & 0.995 & 1.042 & 1.029 & 1.093 & 1.077 & 0.973 \\
\hline VG 62 & 0.998 & 1.029 & 1.029 & 1.097 & 1.048 & 0.984 \\
\hline VG 65 & 1.005 & 1.054 & 1.042 & 1.089 & 0.968 & 1.002 \\
\hline VG 66 & 1.050 & 1.056 & 1.054 & 1.086 & 0.964 & 1.000 \\
\hline VG 69 & 1.035 & 1.072 & 1.058 & 0.966 & 0.957 & 1.051 \\
\hline VG 70 & 0.984 & 1.040 & 1.031 & 1.078 & 0.976 & 1.033 \\
\hline VG 72 & 0.980 & 1.039 & 1.039 & 0.950 & 1.024 & 1.024 \\
\hline VG 73 & 0.991 & 1.028 & 1.015 & 0.972 & 0.965 & 1.013 \\
\hline VG 74 & 0.958 & 1.032 & 1.029 & 0.971 & 1.073 & 1.012 \\
\hline VG 76 & 0.943 & 1.005 & 1.021 & 0.960 & 1.030 & 1.000 \\
\hline VG 77 & 1.006 & 1.038 & 1.026 & 1.078 & 1.027 & 1.004 \\
\hline VG 80 & 1.040 & 1.049 & 1.038 & 1.078 & 0.985 & 1.005 \\
\hline VG 85 & 0.985 & 1.024 & 1.024 & 0.956 & 0.983 & 1.012 \\
\hline VG 87 & 1.015 & 1.019 & 1.022 & 0.964 & 1.088 & 1.013 \\
\hline VG 89 & 1.000 & 1.074 & 1.061 & 0.974 & 0.989 & 1.040 \\
\hline VG 90 & 1.005 & 1.045 & 1.032 & 1.024 & 0.990 & 1.030 \\
\hline VG 91 & 0.980 & 1.052 & 1.033 & 0.961 & 1.028 & 1.002 \\
\hline VG 92 & 0.990 & 1.058 & 1.038 & 0.956 & 0.976 & 1.030 \\
\hline VG 99 & 1.010 & 1.023 & 1.037 & 0.954 & 1.088 & 1.005 \\
\hline VG 100 & 1.017 & 1.049 & 1.037 & 0.958 & 0.961 & 1.001 \\
\hline VG 103 & 1.010 & 1.045 & 1.031 & 1.016 & 0.961 & 1.010 \\
\hline VG 104 & 0.971 & 1.035 & 1.031 & 1.099 & 1.012 & 1.001 \\
\hline VG 105 & 0.965 & 1.043 & 1.029 & 0.969 & 1.068 & 1.050 \\
\hline VG 107 & 0.952 & 1.023 & 1.008 & 0.965 & 0.976 & 1.016 \\
\hline VG 110 & 0.973 & 1.032 & 1.028 & 1.087 & 1.041 & 1.015 \\
\hline VG 111 & 0.980 & 1.035 & 1.039 & 1.037 & 0.979 & 1.007 \\
\hline VG 112 & 0.975 & 1.018 & 1.019 & 1.092 & 0.976 & 1.091 \\
\hline VG 113 & 0.993 & 1.043 & 1.027 & 1.075 & 0.972 & 1.000 \\
\hline VG 116 & 1.005 & 1.011 & 1.031 & 0.959 & 1.033 & 1.006 \\
\hline Tар 8 & 0.985 & 0.999 & 0.994 & 1.011 & 1.004 & 0.942 \\
\hline Tap 32 & 0.960 & 1.017 & 1.013 & 1.090 & 1.060 & 1.004 \\
\hline Tap 36 & 0.960 & 0.994 & 0.997 & 1.003 & 1.000 & 0.956 \\
\hline Tap 51 & 0.935 & 0.998 & 1.000 & 1.000 & 1.000 & 0.930 \\
\hline Tap 93 & 0.960 & 1.000 & 0.997 & 1.008 & 0.992 & 1.001 \\
\hline Tap 95 & 0.985 & 0.995 & 1.020 & 1.032 & 1.007 & 0.972 \\
\hline Tар 102 & 0.935 & 1.024 & 1.004 & 0.944 & 1.061 & 1.004 \\
\hline Tар 107 & 0.935 & 0.989 & 1.008 & 0.906 & 0.930 & 0.942 \\
\hline Tap 127 & 0.935 & 1.010 & 1.009 & 0.967 & 0.957 & 1.001 \\
\hline QC 34 & 0.140 & 0.049 & 0.048 & 0.093 & 0.117 & 0.005 \\
\hline QC 44 & 0.100 & 0.026 & 0.026 & 0.093 & 0.098 & 0.020 \\
\hline QC 45 & 0.100 & 0.196 & 0.197 & 0.086 & 0.094 & 0.161 \\
\hline QC 46 & 0.100 & 0.117 & 0.118 & 0.089 & 0.026 & 0.120 \\
\hline QC 48 & 0.150 & 0.056 & 0.056 & 0.118 & 0.028 & 0.043 \\
\hline QC 74 & 0.120 & 0.120 & 0.120 & 0.046 & 0.005 & 0.110 \\
\hline QC 79 & 0.200 & 0.139 & 0.140 & 0.105 & 0.148 & 0.105 \\
\hline QC 82 & 0.200 & 0.180 & 0.180 & 0.164 & 0.194 & 0.150 \\
\hline QC 83 & 0.100 & 0.166 & 0.166 & 0.096 & 0.069 & 0.122 \\
\hline QC 105 & 0.200 & 0.189 & 0.190 & 0.089 & 0.090 & 0.150 \\
\hline QC 107 & 0.060 & 0.128 & 0.129 & 0.050 & 0.049 & 0.132 \\
\hline QC 110 & 0.060 & 0.014 & 0.014 & 0.055 & 0.022 & 0.001 \\
\hline PG(MW) & 4374.8 & 4359.3 & 4361.4 & $\mathrm{NR}^{*}$ & $\mathrm{NR}^{*}$ & 4362.02 \\
\hline QG(MVAR) & 795.6 & 604.3 & 653.5 & $* \mathrm{NR}^{*}$ & $\mathrm{NR}^{*}$ & 610.11 \\
\hline $\begin{array}{l}\text { Reduction in } \\
\text { PLOSS }(\%)\end{array}$ & 0 & 11.7 & 10.1 & 0.6 & 1.3 & 14.15 \\
\hline Total PLOSS (Mw) & 132.8 & 117.19 & 119.34 & 131.99 & 130.96 & 114.005 \\
\hline
\end{tabular}

NR* - Not reported.

Then IEEE 300 bus system [18] is used as test system to validate the performance of Enhanced Wormhole Optimizer (EWO) algorithm. Table 12 shows the comparison of real power loss obtained after optimization.

Table 12. Comparison of Real Power Loss

\begin{tabular}{ccccc}
\hline Parameter & Method CSA [20] & Method EGA [21] & Method EEA [21] & EWO \\
\hline PLOSS (MW) & 635.8942 & 646.2998 & 650.6027 & 612.1026 \\
\hline
\end{tabular}




\section{CONCLUSION}

In this paper proposed Enhanced Wormhole Optimizer (EWO) algorithm successfully solved the optimal reactive power problems. Between different universes objects are exchanged through white or black hole tunnels. Regardless of the inflation rate, through wormholes objects in all universes which possess high probability will shift to the most excellent universe. In between two universes wormhole tunnel are built then the local change for each universe is done by most excellent universe and the elevated probability of refinement the inflation rate through wormholes. Levy flight has been applied effectively and it leads to the improvement of the quality of solution. Proposed Enhanced Wormhole Optimizer (EWO) algorithm has been tested in standard IEEE 14, 30, 57,118,300 bus test systems and simulation results show that the EWO algorithm reduced the real power loss efficiently. Percentage of real power loss reduction has been enhanced when compared to other standard algorithms.

\section{REFERENCES}

[1] K. Y. Lee, "Fuel-cost minimisation for both real and reactive-power dispatches," Proceedings Generation, Transmission and Distribution Conference, vol. 131, no. 3, pp. 85-93, 1984.

[2] N. I. Deeb, "An efficient technique for reactive power dispatch using a revised linear programming approach," Electric Power System Research, vol. 15, no. 2, pp. 121-134, 1998.

[3] M. R. Bjelogrlic, M. S. Calovic, B. S. Babic, "Application of Newton's optimal power flow in voltage/reactive power control," IEEE Trans Power System, vol. 5, no. 4, pp. 1447-1454, 1990.

[4] S. Granville, "Optimal reactive dispatch through interior point methods," IEEE Transactions on Power System, vol. 9, no. 1, pp. 136-146, 1994.

[5] N. Grudinin, "Reactive power optimization using successive quadratic programming method," IEEE Transactions on Power System, vol. 13, no. 4, pp. 1219-1225, 1998.

[6] Ng Shin Mei, R., Sulaiman, M.H., Mustaffa, Z., Daniyal, H., "Optimal reactive power dispatch solution by loss minimization using moth-flame optimization technique," Appl. Soft Comput., vol. 59, pp. 210-222, 2017.

[7] Chen, G., Liu, L., Zhang, Z., Huang, S., "Optimal reactive power dispatch by improved GSA-based algorithm with the novel strategies to handle constraints," Appl. Soft Comput., vol. 50, pp. 58-70, 2017.

[8] Roy, Provas Kumar and Susanta Dutt, "Economic load dispatch: Optimal power flow and optimal reactive power dispatch concept," Optimal Power Flow Using Evolutionary Algorithms, IGI Global, pp. 46-64, 2019.

[9] Christian Bingane, Miguel F. Anjos, Sébastien Le Digabel, "Tight-and-cheap conic relaxation for the optimal reactive power dispatch problem," IEEE Transactions on Power Systems, vol. 34, no. 6, 2019.

[10] Dharmbir Prasad \& Vivekananda Mukherjee, "Solution of Optimal Reactive Power Dispatch by Symbiotic Organism Search Algorithm Incorporating FACTS Devices," IETE Journal of Research, vol. 64, no. 1, pp. 149-160, 2018.

[11] TM Aljohani, et al, "Single and multiobjective optimal reactive power dispatch based on hybrid artificial physicsparticle swarm optimization," Energies, vol. 12, no. 12, p. 2333, 2019

[12] Ram Kishan Mahate, \& Himmat Singh, "Multi-Objective Optimal Reactive Power Dispatch Using Differential Evolution," International Journal of Engineering Technologies and Management Research, vol. 6, no. 2, pp. 27-38, 2019.

[13] Yalçın, E, Taplamacıŏlu, M., Çam, E., "The Adaptive Chaotic Symbiotic Organisms Search Algorithm Proposal for Optimal Reactive Power Dispatch Problem in Power Systems," Electrica, vol. 19, no. 1, pp. 37-47, 2019.

[14] Mouassa, S. and Bouktir, T., "Multi-objective ant lion optimization algorithm to solve large-scale multi-objective optimal reactive power dispatch problem," COMPEL - The international journal for computation and mathematics in electrical and electronic engineering, vol. 38, no. 1, pp. 304-324, 2018.

[15] Tawfiq M. Aljohani, Ahmed F. Ebrahim, Osama Mohammed, "Single and multiobjective optimal reactive power dispatch based on hybrid artificial physics-particle swarm optimization,” Energies, vol. 12, no. 12, pp. 2333, 2019.

[16] Abdechiri M, Meybodi MR and Bahrami H., "Gases Brownian motion optimization: An algorithm for optimization (GBMO),” Applied Soft Computing, vol. 13, no. 5, pp. 2932-2946, 2013.

[17] Mirjalili SM and Hatamlou A., "Multi-verse optimizer: A nature-inspired algorithm for global optimization," Neural Computing and Applications, vol. 27, no. 2, pp. 495-513, 2016.

[18] IEEE, "The IEEE-test systems," 1993. http://www.ee.washington.edu/trsearch/pstca/.

[19] Ali Nasser Hussain, Ali Abdulabbas Abdullah and Omar Muhammed Neda, "Modified particle swarm optimization for solution of reactive power dispatch," Research Journal of Applied Sciences, Engineering and Technology, vol. 15, no. 8, pp. 316-327, 2018.

[20] S. Surender Reddy, "Optimal reactive power scheduling using cuckoo search algorithm," International Journal of Electrical and Computer Engineering (IJECE), vol. 7, no. 5, pp. 2349-2356. 2017.

[21] S.S. Reddy, et al., "Faster evolutionary algorithm based optimal power flow using incremental variables," Electrical Power and Energy Systems, vol. 54, pp. 198-210, 2014. 